\title{
Fístula dural intracraniana com drenagem perimedular: relato de caso
}

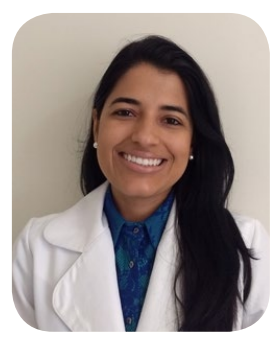

\author{
Juliana de Oliveira Nunes ${ }^{1}$, Carolina Salles de Andrade Carrera ${ }^{1}$, \\ Marcel Leal Ribeiro ${ }^{1}$, Cristiane Abbehusen Lima², Márcio Azevedo \\ de Andrade $^{3}$, Pedro Antônio Pereira de Jesus ${ }^{1}$
}

\section{INTRODUÇÃO}

As fístulas arteriovenosas durais intracranianas (FAVDS) são shunts patológicos entre as artérias durais e os seios venosos durais, veias meníngeas ou veias corticais. As FAVDS representam cerca de $10 \%$ a $15 \%$ de todas as malformações arteriovenosas, acometendo principalmente a faixa etária entre 50 e 60 anos $^{1,2}$. Essas lesões apresentam predomínio em pessoas do sexo masculino, com uma proporção relativa ao sexo feminino de 4:12.

As FAVDS podem ser espontâneas, idiopáticas ou adquiridas. Elas podem ter associação com traumatismo craniano, otite, trombose de seio cavernoso, craniotomia prévia e gravidez ${ }^{2}$. Em 1982, Woimant et. al. descreveram o primeiro caso de FAVDS com drenagem perimedular e até o momento há menos de 100 casos descritos na literatura ${ }^{3}$. Descrevemos um relato de caso de um paciente admitido no hospital Santa Izabel sem diagnóstico e que após investigação foi confirmada FAVDS.

PALAVRAS-CHAVE: fístula dural arteriovenosa; cognard tipo $\mathrm{V}$; angiografia

KEY-WORDS: duralarteriovenous fistula; cognardtype V; angiography

\section{RELATO DE CASO}

Paciente do sexo masculino, 70 anos, natural e procedente de Salvador-Bahia, destro, aposentado e com diagnóstico prévio de hipertensão arterial iniciou há 6 meses da admissão hospitalar quadro clínico caracterizado inicialmente por parestesias em membros inferiores que evoluiu com piora progressiva, acometendo também membros superiores, sendo mais intensos em mãos e pés. Durante o período, o paciente apresentou queixa de dor na região dorsal e, após cinco meses do início dos sintomas, iniciou episódios de incontinência urinária. Negou quadro infeccioso antecedente ao início dos sin- tomas ou história prévia de traumas. O paciente realizou inicialmente investigação ambulatorial e entre os exames solicitados a eletroneuromiografia demonstrava neuropatia sensitivo-motora axonal com predomínio sensorial em membros inferiores. O estudo do líquor evidenciava dissociação proteíno-citológica e a ressonância magnética (RM) de crânio, coluna torácica e lombar sem contraste não apresentaram alterações significativas. O paciente, então, foi encaminhado ao Hospital Santa Izabel para elucidação diagnóstica, pois os exames acima realizados não evidenciaram possível etiologia.

$\mathrm{Na}$ admissão hospitalar, ao exame físico neurológico o paciente encontrava-se: vígil, lúcido e orientado em tempo e espaço, fala e linguagem sem alterações. Pupilas isocóricas e fotorreagentes, pares cranianos preservados. Força grau 4 em membros inferiores, força nos membros superiores estava preservada. Apresentava hipoestesia tátil em membros com padrão bota e luva, além de nível sensitivo em T3. Reflexos tendíneos profundos estavam exacerbados em membros inferiores com presença de clônus esgotável. Os demais sistemas não apresentavam alterações significativas.

Os exames laboratoriais iniciais não apresentaram alterações. Foram realizadas novas RM das colunas cervical, torácica e lombar, com contraste venoso, que elucidaram o diagnóstico, demonstrando proeminência de vasos na superfície meníngea da medula espinhal, sobretudo da torácica e em torno do cone medular, considerando como um sinal indireto da presença de uma fístula dural, indicando a necessidade de estudo angiográfico complementar para determinar a localização da fístula propriamente dita (Figura 1). A RM da coluna torácica também demonstrou alguns pequenos focos periféricos de hipersinal na ponderação T2, podendo corresponder a focos de edema ou pequenos infartos na medula. (Figura 1). A realização da RM de coluna com contraste foi elucidativa para o diagnóstico, guiando a investigação, sendo 
optado em realizar arteriografia para melhor investigação dos vasos medulares e cerebrais.

$\mathrm{O}$ paciente foi submetido à arteriografia cerebral e medular, sendo observado fístula dural de alto fluxo (Figura 2) com ramos originados de vários ramos da artéria carótida externa esquerda, fluindo para o seio lateral esquerdo com drenagem da veia perimedular, além de artéria aferente da artéria meníngea posterior esquerda (classificação Cognard tipo V). Após realização da arteriografia, paciente recebeu alta hospitalar com programação de nova abordagem após algumas semanas. Em um segundo momento, foi realizada nova abordagem endovascular, sendo utilizado polímero de etileno vinil álcool (Onyx) para embolização da fístula. Após procedimento, paciente apresentou melhora significativa da força em membros inferiores, mas manteve quadro recorrente de incontinência urinária.

\section{DISCUSSÃO}

A classificação de Cognard para as fístulas durais intracranianas é feita de acordo com a localização da fístula, presença de drenagem venosa cortical, direção do fluxo e a presença de ectasia venosa. Essa classificação é graduada de I a V e o tipo Cognard V se caracteriza por drenagem venosa para veias perimedulares. As fístulas tipo I são benignas e as do tipo II ao V são indicadas tratamento ${ }^{3,4}$.

O quadro clínico dos pacientes com fístula Cognard tipo V se caracteriza por uma mielopatia ascendente, disfunções esfincterianas ou sintomas bulbares que podem estar distantes do local da fístula ${ }^{5}$. Os sintomas geralmente se desenvolvem lentamente por semanas ou meses, de acordo a congestão venosa do sistema perimedular. Entretanto, alguns relatos de casos apontam para uma progressão rapidamente progressiva para uma completa tetraparesia em alguns dias.

O diagnóstico desse tipo de fístula, baseado apenas em critérios clínicos, pode se tornar desafiante, já que diversos outros diagnósticos podem mimetizar patologias medulares, tais como tumores, isquemia, Guillain-Barré ou esclerose múltipla ${ }^{4,5}$. Na ressonância magnética da medula, entre as alterações mais sugestivas podem ser vistas hipointensidade intramedular e flow voids na superfície medular. Na sequência com contraste são visualizados vasos serpentiformes com realce na superfície medular sugestiva de ectasias vasculares. No paciente em questão, a ressonância magnética de coluna torácica com contraste foi elucidativa para o diagnóstico, pois mostrou alterações compatíveis com FAVDS, direcionando, assim, o tratamento. A angiografia por subtração digital é o exame padrão ouro que permite identificar arté- rias nutridoras da fístula, a localização e fluxo da FAVDS, além da drenagem venosa ${ }^{4}$.

O objetivo do tratamento das FAVDS é eliminar a congestão venosa da medula espinhal, preservando a drenagem venosa antes que ocorra uma alteração irreversível. A abordagem via endovascular tornou-se o principal manejo para tratamento das FAVDS, reservando a abordagem cirúrgica intracraniana para os casos em que a embolização não tenha sucesso ou apresente recorrência do quadro clínico. Alguns materiais podem ser utilizados na embolização, tais como Onyx e n-butilcianoacrilato. Nesse relato de caso, foi utilizado para a embolização da FAVDS o material Onyx, um agente embólico não adesivo, composto por etileno e álcool vinílico dissolvido em dimetilsulfóxido ${ }^{6}$. Artigos recentes demonstraram que a técnica endovascular utilizando Onyx tem algumas vantagens sobre o cianoacrilato, incluindo um maior controle do processo embólico do agente durante a injeção e tempo de injeção com poucas complicações ${ }^{7}$.

Caso a FAVDS não seja tratada em tempo hábil, dados da literatura refletem elevada morbidade e mortalidade relacionada à doença. Cerca de $30 \%$ dos pacientes morrem ou permanecem inalterados a despeito do tratamento; $18 \%$ apresentam pequena melhora, permanecendo incapacitados; e 52\% apresentam melhora substancial, evoluindo sem déficit ou com déficit mínimo ${ }^{8}$. O paciente relatado apresentou melhora significativa da força em membros inferiores, manteve episódios recorrentes de incontinência urinária e continuou independente para a realização das suas atividades diárias.

Esse relato de caso demonstra uma patologia rara com apresentação clínica variada, que deve fazer parte do diagnóstico diferencial das mielopatias. A realização de um diagnóstico precoce é substancial para proporcionar um tratamento eficaz e melhor desfecho clínico.

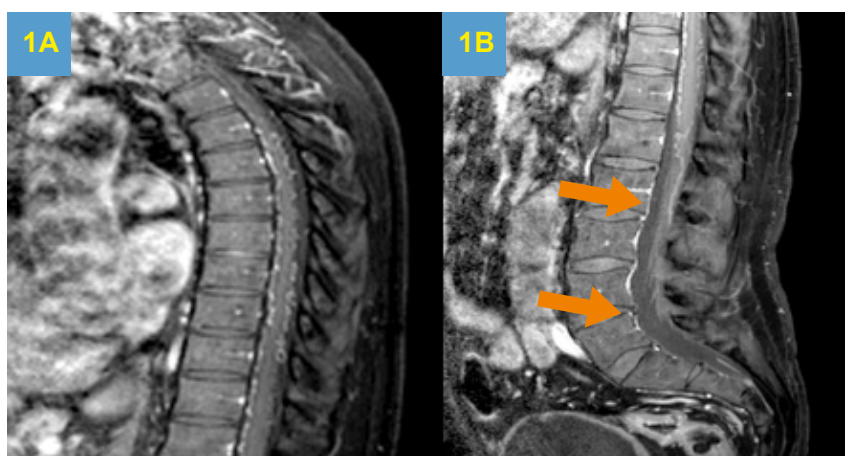

Figura 1 - Ressonância magnética da coluna torácica (A) e lombar (B) na fase pós-contraste, demonstrando vasos piais tortuosos e ectasiados (setas), representando congestão venosa 


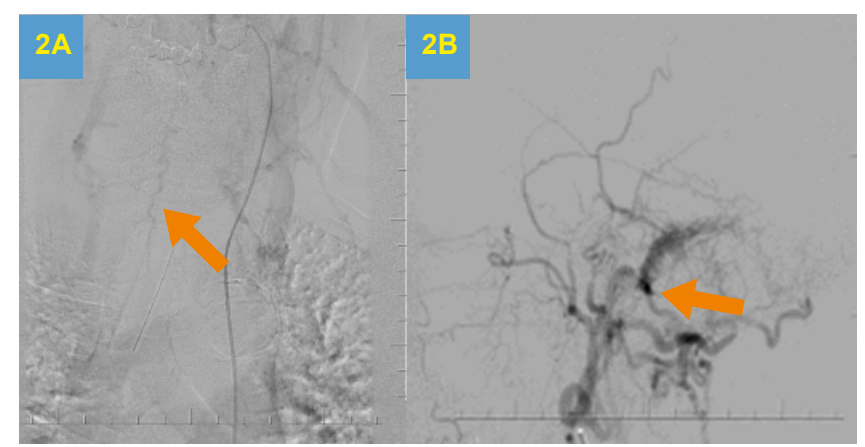

Figura 2 - Arteriografia mostrando drenagem para a veia perimedular $(A)$ e seio lateral esquerdo $(B)$

\section{REFERÊNCIAS}

1. Jermakowicz WJ, Weil AG, Vlasenko A, Bhatia $S$, Niazi TN. CognardType V intracranialduralarteriovenous fistula presenting in a pediatricpatientwithrapid, progressivemyelopathy. J NeurosurgPediatr. 2017;20(2):158-63.

2. Sugiura Y, Nozaki T, Sato H, Sawashita K, Hiramatsu $\mathrm{H}$, Nishizawa $\mathrm{S}$. Sigmoid sinus dural arteriovenous fistula with spinal venous drainage manifesting as only brain stem-related neurological deficits without myelopathy: case report. Neurol Med Chir (Tokyo). 2009;49(2):71-76.

3. El Asri AC, El Mostarchid B, Akhaddar A, Naama O, Gazzaz M, Boucetta M. Factors Influencing the Prognosis in Intracranial Dural Arteriovenous Fistulas with Perimedullary Drainage. World Neurosurgery. 2013;79(1):182-91.

4. Signorelli F, Gory B, Maduri R, Guyotat J, Pelissou-Guyotat I, Chirchiglia D, et. al. Intracranial Dural Arteriovenous Fistulas: A Review. Journal of Neurology \& Stroke. 2014;1(2):1-0.

5. Chaichana KL, Coon AL, Tamargo RJ, Huang J. DuralArteriovenous Fistulas: EpidemiologyandClinicalPresentation. NeurosurgClin N Am. 2012;23(1):7-13.

6. Carvalho JC, Machin FJ, Manzanera LS, et. al. Intraventricular hemorrhageafterdural fistula embolization. Braz J Anesthesiol 2017; 67: 199-204.

7. Trivelato FP, Abud DG, Ulhôa AC, Menezes T de J, Abud TG, Nakiri GS, et. al. Duralarteriovenous fistulas withdirect cortical venousdrainagetreatedwithOnyx ${ }^{\circledR}$ : a case series. Arquivos de Neuro-Psiquiatria. 2010;68(4):613-8.

8. Seda JR, Lauro de Franco et. al. Fístula arteriovenosa dural intracraniana com drenagem perimedular: relato de caso. Arq. Neuro-Psiquiatr., São Paulo, v. 60, n. 3B, p. 856-860, 2002.
1- Serviço de Neurologia do HSI

2- Serviço de Neurorradiologia do HSI

3- Serviço de Neurorradiologia Intervencionista do HSI

Endereço para correspondência:

onunes.ju@gmail.com 